\title{
Status of the instrumentation program for the Giant Magellan Telescope
}

\author{
George H. Jacoby*a, A. Bouchez ${ }^{\mathrm{a}}$, M. Colless ${ }^{\mathrm{b}}$, D. DePoy ${ }^{\mathrm{c}}$, D. Jaffe ${ }^{\mathrm{d}}$, J. Lawrence ${ }^{\mathrm{e}}$, P. McGregor ${ }^{\mathrm{b}}$, \\ R. Bernstein ${ }^{\mathrm{a}, \mathrm{f}}$, S. Shectman ${ }^{\mathrm{f}}$, A. Szentgyorgyi ${ }^{\mathrm{g}}$ \\ ${ }^{\mathrm{a}}$ Giant Magellan Telescope Organization, 251 S. Lake Ave, Pasadena, CA 91101; ${ }^{\mathrm{b}}$ Research School \\ of Astronomy and Astrophysics, The Australian National University, Cotter Road, Weston, ACT, \\ 2611, Australia; ' Department of Physics and Astronomy, Texas A\&M University, College Station, \\ TX 77843; ${ }^{\mathrm{d}}$ Department of Astronomy, University of Texas at Austin, Austin, TX; ${ }^{\mathrm{e}}$ Australian \\ Astronomical Observatory, PO Box 296 Epping, NSW 1710, Australia; ${ }^{\mathrm{f} C a r n e g i e}$ Observatories, 813 \\ Santa Barbara St., Pasadena, CA; ${ }^{g}$ Harvard-Smithsonian Center for Astrophysics, 60 Garden St., \\ Cambridge, MA 0213891101
}

\begin{abstract}
Instrument development for the $25 \mathrm{~m}$ class optical/infrared Giant Magellan Telescope (GMT) is actively underway. Two instruments have begun their preliminary design phase: an optical (350-1000 nm) high resolution and precision radial velocity echelle spectrograph (G-CLEF), and a near-IR (YJHK) diffraction-limited imager/integral-field-spectrograph (GMTIFS). A third instrument will begin its design phase in early 2015: an optical (370-1000 nm) low-to-medium resolution multi-object spectrograph (GMACS). Two other instrument teams are focusing on prototypes to demonstrate final feasibility: a near-to-mid-IR (JHKLM) high resolution diffraction-limited echelle (GMTNIRS) spectrograph, and a facility robotic multi-fiber-feed (MANIFEST). A brief overview of the GMT instrumentation program is presented: current activities, progress, status, and schedule, as well as a summary of the facility infrastructure needed to support the instruments.
\end{abstract}

Keywords: GMT, ELT, spectrograph, integral-field spectrograph, multi-object spectrograph, optical instruments, infrared instruments, adaptive optics, imaging, gratings

\section{INTRODUCTION}

The selection of first-generation instruments for the Giant Magellan Telescope (GMT) was completed in 2012. The design and development of each instrument is underway. The cadence and sequencing for this phase of the instrumentation program is driven by the technical status of the individual instrument designs, the funding profile available to the program, and the system-wide readiness of the infrastructure needed by the different instruments (e.g., adaptive secondary mirrors, laser guide stars, wide-field corrector).

Each instrument has been developed through a conceptual design, as reported at the previous SPIE meeting by Jacoby ${ }^{1}$. Since then, the construction plan for the entire GMT facility has matured significantly, thereby providing detailed guidance on the availability of instrument funding and the schedule for the facility infrastructure to accommodate each instrument. This report summarizes those plans as well as recent progress on the development of each instrument since the 2012 report.

We also provide a brief description of each instrument, its science drivers, and where it stands in the GMT commissioning plan. Detailed descriptions for all the instrument designs are presented elsewhere in this conference or the previous one.

* gjacoby@gmto.org; phone: +1 520 904-4135; www.gmto.org

Ground-based and Airborne Instrumentation for Astronomy V, edited by

Suzanne K. Ramsay, lan S. McLean, Hideki Takami, Proc. of SPIE Vol. 9147,

91471Y · (C) 2014 SPIE · CCC code: 0277-786X/14/\$18 · doi: 10.1117/12.2055487

Proc. of SPIE Vol. 9147 91471Y-1 
For the purposes of the GMT, the roles of instruments are broadly defined to be:

- Detect the photons collected by the primary mirror, within the wavelength range available to the GMT.

- Convert those photons into a form that can be saved and later analyzed to yield information about their source, or about the environment(s) between the source and the instrument.

Table 1 summarizes key GMT parameters that serve as a foundation for instrument development. Overviews of the facility and the telescope are presented elsewhere at this meeting ${ }^{2,3}$.

Table 1. Key GMT telescope parameters for instruments

\begin{tabular}{|c|c|}
\hline Parameter & Value \\
\hline Aperture largest diameter & $25.4 \mathrm{~m}$ \\
\hline M1 f/ratio & 0.71 \\
\hline Aperture, collecting area & $368-\mathrm{m}^{2}$ \\
\hline Final f/ratio, narrow- / wide-field & $8.16 / 8.34$ \\
\hline Unvignetted field of view, narrow / wide & $10 / 20^{\mathrm{a}} \operatorname{arcmin}$ (diameter) \\
\hline Number of reflections, $\mathrm{AO} /$ natural & $3 / 2$ \\
\hline Plate scale, narrow- / wide-field & $0.994 / 0.972 \mathrm{arcsec} / \mathrm{mm}$ \\
\hline $50^{\text {th }}$ percentile seeing (V-band), FWHM & $0.63 \operatorname{arcsec}$ \\
\hline Adaptive optics correction & Adaptive M2 (ASM) \\
\hline Strehl $^{b}$, JHK, NGSAO & $0.40 / 0.60 / 0.75 \quad(\mathrm{~V}=8, \mathrm{G} 2 \mathrm{~V}$ star $)$ \\
\hline Strehl $^{\mathrm{b}}, \mathrm{JHK}, \mathrm{LTAO}$ & $0.12 / 0.30 / 0.50$ \\
\hline $\mathrm{FWHM}^{\mathrm{b}}$, GLAO RIJHK & $0.46 / 0.43 / 0.34 / 0.28 / 0.23 \operatorname{arcsec}$ \\
\hline FWHM $^{\mathrm{b}}$, Natural seeing RIJHK & $0.55 / 0.52 / 0.46 / 0.41 / 0.37 \operatorname{arcsec}$ \\
\hline Telescope location & Las Campanas, Chile \\
\hline
\end{tabular}

${ }^{a}$ Wide-field operation suffers slight vignetting from the active optics wavefront sensors

${ }^{\mathrm{b}}$ Measured at a zenith angle of $15 \mathrm{deg}$, assuming median conditions and wind speed of $6.3 \mathrm{~m} / \mathrm{s}$

\section{STAGED IMPLEMENTATION OF THE GMT FACILITY}

The GMT facility will be commissioned over a period of several years, bringing capabilities and features on-line over this period. Several factors favor a staged implementation plan.

- Expenditures for major purchases can be optimally matched to the available funding profile

- Long-lead items (e.g., primary mirror segments; adaptive secondary mirrors) require a delivery schedule that extends beyond the earliest opportunity to begin science operations

- Commissioning strategies follow a natural progression (e.g., commission natural seeing modes before AOcorrected modes), thereby permitting some components to be deferred for later delivery. Also, observatory personnel can more easily focus on a few major activities over the longer implementation time instead of scheduling many activities in a short time, thereby reducing contention for access to the facility.

The GMT plan is separated into three overlapping stages spanning $\sim 4$ years. The key capabilities that will be addressed in each stage are summarized in Table 2 . The instrument delivery schedules are tied to these stages. 
Table 2. Key Capabilities of the GMT Implementation Stages

\begin{tabular}{|c|c|}
\hline Stage & Key Capabilities \\
\hline 1 & $\begin{array}{l}4 \text { Primary Mirror Segments } \\
4 \text { Fast Steering Secondary Mirror Segments } \\
\text { Natural Seeing Instrument: G-CLEF (high resolution optical spec; } R=\mathbf{2 0 K - 1 0 0 K )} \\
\text { Natural Seeing Instrument: GMACS (low-medium resolution optical MOS; } \mathbf{R} \sim \mathbf{3 K} \text { ) }\end{array}$ \\
\hline 2 & $\begin{array}{l}7 \text { Primary Mirror Segments } \\
4 \text { Adaptive Secondary Mirror Segments; } 7 \text { Fast Steering Mirror Segments } \\
\text { Natural Guide Star AO } \\
\text { Ground Layer AO } \\
\text { AO Instrument: GMTIFS (High Strehl IFU, R=5K-10K; AO imager) }\end{array}$ \\
\hline 3 & $\begin{array}{l}7 \text { Primary Mirror Segments } \\
7 \text { Adaptive Secondary Mirror Segments; } 7 \text { Fast Steering Mirror Segments } \\
\text { Laser Guide Star AO } \\
\text { Wide Field (20') Corrector/ADC } \\
\text { AO Instrument: GMTNIRS (Medium Strehl JHKLM echelle; R=50K-100K) } \\
\text { Wide Field Robotic Fiber Positioner (MANIFEST, feeds G-CLEF \& GMACS) }\end{array}$ \\
\hline
\end{tabular}

\section{INSTRUMENT PROGRAM OVERVIEW}

Five primary instrument concepts were well advanced by the time of the GMT System CoDR in 2006. The conceptual properties for two of these (GMTNIRS and GMACS) are retained in the current instrument suite. Jacoby ${ }^{1}$ described the selection process that has led from the original five instruments to seven candidates, and finally, to the five instruments under development today. Like other large telescope projects, defining and finalizing the instrument selection is a dynamical process.

The high level characteristics of these five instruments are given in Table 3.

Table 3. The five instruments that are currently under development by GMT. MANIFEST is not strictly an instrument because it is only provides a fiber feed to other instruments and does not detect light. Primarily it feeds G-CLEF to convert it to a multi-object spectrograph, and GMACS to provide it with alternatives to slitlets while improving the spectral resolution up to 10,000. The AO Modes are Natural Seeing (NS), Ground Layer AO (GLAO), Natural Guide Star AO (NGSAO), and Laser Tomography AO (LTAO), where secondary modes are shown in grey.

\begin{tabular}{|l|c|l|c|c|l|}
\hline Instrument & AO Modes & \multicolumn{1}{c|}{ Function } & $\lambda$ Range, $\mu$ m & Resolution & \multicolumn{1}{c|}{ Field of View } \\
\hline G-CLEF & $\begin{array}{c}\text { NS, GLAO, } \\
\text { NGSAO }\end{array}$ & $\begin{array}{l}\text { Optical High Resolution } \\
\text { Spectrometer / PRV }\end{array}$ & $0.35-1.0$ & $20,000-100,000$ & $\begin{array}{l}7 \times 0.7 ”, \& \\
7 \times 1 " \text { fibers }\end{array}$ \\
\hline GMACS & NS, GLAO & $\begin{array}{l}\text { Wide-Field Optical Multi- } \\
\text { Object Spectrometer }\end{array}$ & $0.37-1.0$ & $1,500-4,000$ & $40-60 \mathrm{arcmin}^{2}$ \\
\hline GMTIFS & $\begin{array}{c}\text { LTAO, } \\
\text { NGSAO }\end{array}$ & NIR AO-fed IFU / Imager & $0.9-2.5$ & $5,000 \& 10,000$ & $10 / 400 \mathrm{arcsec}^{2}$ \\
\hline GMTNIRS & $\begin{array}{c}\text { NGSAO, } \\
\text { LTAO }\end{array}$ & $\begin{array}{l}\text { JHKLM AO-fed High } \\
\text { Resolution Spectrometer }\end{array}$ & $1.15-5.3$ & $\begin{array}{c}50,000 / 100,000 \\
\text { (JHK / LM) }\end{array}$ & 1.2 " long-slit \\
\hline MANIFEST & NS, GLAO & Facility Robotic Fiber Feed & $0.36-1.0$ & & 20 arcmin diam \\
\hline
\end{tabular}




\subsection{Overview of the Instrument Development Status}

During the period 2010-2011, each of the instruments was developed to a conceptual design level and taken through a design review (CoDR). The exception was MANIFEST because it required technologies that did not exist at that time; instead, the MANIFEST instrument team carried out a series of feasibility studies to prepare for a later conceptual design currently planned for 2016-2017.

At this time, GMT has contracted with all five instrument teams to advance their designs further. The G-CLEF and GMTIFS designs were well developed at the time of their CoDR and those teams have been directed to proceed through a preliminary design. The remaining three instrument teams are working toward shorter-term goals appropriate for their level of readiness. Table 4 summarizes the activities for each of the instruments and the completion date for the current phase.

Table 4. The current activities and next planned activities for each of the instruments.

\begin{tabular}{|l|l|l|l|c|}
\hline Instrument & \multicolumn{1}{|c|}{$\begin{array}{c}\text { Principal } \\
\text { Investigator }\end{array}$} & Lead Institution & Current / Next Activity & $\begin{array}{c}\text { Completion } \\
\text { Date for } \\
\text { Current } \\
\text { Activity }\end{array}$ \\
\hline G-CLEF & A. Szentgyorgyi & $\begin{array}{c}\text { Smithsonian } \\
\text { Astrophysical } \\
\text { Observatory }\end{array}$ & $\begin{array}{l}\text { Preliminary design, prototyping / final } \\
\text { design and fabrication }\end{array}$ & Mid 2015 \\
\hline GMACS & D. DePoy & $\begin{array}{c}\text { Texas A \& M } \\
\text { University }\end{array}$ & $\begin{array}{l}\text { Trade studies to define instrument } \\
\text { functional properties / incremental } \\
\text { conceptual design }\end{array}$ & Late 2014 \\
\hline GMTIFS & P. McGregor & $\begin{array}{c}\text { Australian National } \\
\text { University }\end{array}$ & $\begin{array}{l}\text { Preliminary design, prototyping / final } \\
\text { design and fabrication }\end{array}$ & Late 2016 \\
\hline GMTNIRS & D. Jaffe & $\begin{array}{c}\text { University of Texas, } \\
\text { Austin }\end{array}$ & $\begin{array}{l}\text { Optical design, grating development } \\
\text { and manufacture / preliminary design }\end{array}$ & Late 2016 \\
\hline MANIFEST & $\begin{array}{l}\text { M. Colless } \\
\text { J. Lawrence }\end{array}$ & $\begin{array}{c}\text { Australian } \\
\text { Astronomical } \\
\text { Observatory }\end{array}$ & $\begin{array}{l}\text { Prototyping fiber positioners, fibers, } \\
\text { field plate, electronics, and on-sky } \\
\text { demonstrator / conceptual design }\end{array}$ & Early 2015 \\
\hline
\end{tabular}

In preparation for the activities listed in Table 4, each team has generated a set of science drivers in order to specify the science requirements and goals for their instruments. The science requirements then flow down to the functional, technical, and operational instrument requirements. For the purposes of uniformity and clarity, instrument teams were directed to think of these 3 levels of requirements (science drivers, science requirements, instrument requirements) in terms of an observing proposal in the following sense:

- The science drivers are the overarching goals of the science case, like the introductory paragraphs in an observing proposal (e.g., discovering the most metal-poor stars in the Galaxy).

- The science requirements represent the observational elements of the science justification in an observing proposal (e.g., spectral coverage must include iron lines).

- The instrument requirements represent the technical discussion in an observing proposal, describing the expectations of the equipment to allow measurement of the observational elements described above (e.g., wavelength range from $400-550 \mathrm{~nm}$; resolution $>40,000$ )

- If a science requirement has concepts that specify an instrument parameter (e.g., resolution $>5,000$ ), then it isn't a science requirement. The science requirement defines the observational goal (e.g. resolving the line structure of Lya in QSOs).

These requirements are captured in the GMT requirements tool (Cognition Cockpit) in order to document and validate proper flow down from all systems (e.g., telescope, enclosure, AO, software) to the instrument. 


\subsection{Next Generation Instruments}

Now that the first generation instruments are in development, GMT can begin considering a second generation. The time scales for the end-to-end process are long. A decade is a typical, or even optimistic, life cycle. We will start soon by identifying the conceivable science questions. The GMT Project Scientist will lead the activities with support from the GMT Science Advisory Committee.

\section{BRIEF DESCRIPTION OF THE INSTRUMENTS}

Each instrument team is presenting updates on their instrument development at this meeting. Therefore, this paper provides only a terse summary of each instrument.

\subsection{G-CLEF}

The GMT-Consortium Large Earth Finder (G-CLEF) is scheduled to be the first science instrument at GMT. It is a fiberfed optical $(350-1000 \mathrm{~nm}$, simultaneous $)$ echelle spectrograph that provides the observational capabilities of both a general-purpose high resolution spectrograph and a precision radial velocity spectrograph ${ }^{4}$. G-CLEF will address the following key science areas, but also has application to extragalactic studies in the Local Group as well as distant sources.

- Characterizing the most metal poor stars, such as the $[\mathrm{Fe} / \mathrm{H}]<-7.1$ star SMSS J031300.36-670839. $3^{5}$

- Measuring exoplanet masses, possibly as small as Mars around an M-star

- Finding $\mathrm{O}_{2}$ in exoplanet atmospheres using transmission spectra ${ }^{6}$

The G-CLEF design uses an innovative asymmetric white pupil, dual-beam optical design ${ }^{7}$ (Figure 1) that minimizes aberrations due to field curvature at the detector focal plane. A precision vacuum enclosed thermal control system is necessary to meet the extreme stability requirement that precision velocity measurements $(<50 \mathrm{~cm} / \mathrm{s}$; goal $10 \mathrm{~cm} / \mathrm{s})$ demand, along with resolutions up to 100,000. For more modest observational programs, additional modes are available to re-balance spectral resolution against spectrograph throughput, as summarized in Table 5. Modes are chosen by moving the input fiber selector. G-CLEF can also be fed by the MANIFEST facility wide-field fiber feed to operate in a multi-object (5-40 targets) mode or to offer an IFU capability.

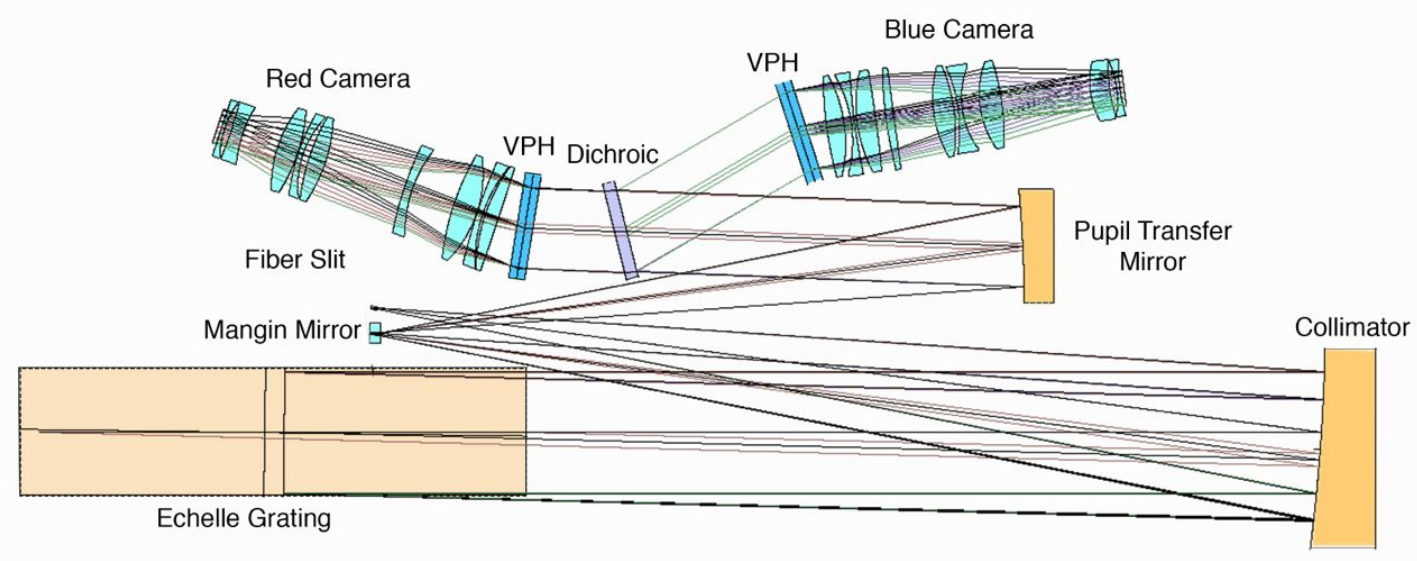

Figure 1. The optical layout of the G-CLEF spectrograph

Like all instruments, G-CLEF benefits from the intrinsic AO capabilities of GMT. With GLAO, the throughput of the PRV modes improves by $\sim 25 \%$, and the HT mode improves by $~ 5 \%$ over the values in Table 5 . Further, G-CLEF can be 
upgraded with higher order wavefront sensors to utilize NGSAO. In that AO mode, the throughput improves by up to a factor of two, and the velocity precision also improves due to the high stability of the input image.

Table 5. Summary of key observing modes for G-CLEF. Peak throughput assumes a Gaussian PSF.

\begin{tabular}{|l|l|c|c|}
\hline Observing Modes & \multicolumn{1}{|c|}{ Description } & $\begin{array}{c}\text { Spectral } \\
\text { Resolution }\end{array}$ & $\begin{array}{c}\text { Peak } \\
\text { Throughput }\end{array}$ \\
\hline $\begin{array}{l}\text { High Throughput } \\
\text { (HT) }\end{array}$ & $\begin{array}{l}\text { Maximum throughput, moderate } \\
\text { resolution }\end{array}$ & 20,000 & $17.9 \%$ \\
\hline $\begin{array}{l}\text { Medium Throughput } \\
\text { (MT) }\end{array}$ & $\begin{array}{l}\text { Throughput and resolution are } \\
\text { balanced }\end{array}$ & 35,000 & $13.2 \%$ \\
\hline $\begin{array}{l}\text { Precision Radial Velocity } \\
\text { (PRV) }\end{array}$ & $\begin{array}{l}\text { Velocity precision is optimized at } \\
\text { maximum resolution }\end{array}$ & 105,000 & $10.2 \%$ \\
\hline $\begin{array}{l}\text { Non-scrambled PRV } \\
\text { (NS-PRV) }\end{array}$ & $\begin{array}{l}\text { Maximum resolution without } \\
\text { scrambling the fiber modes to } \\
\text { improve high throughput }\end{array}$ & 105,000 & $12.3 \%$ \\
\hline $\begin{array}{l}\text { Multi-object Spectrograph } \\
\text { (MOS) }\end{array}$ & $\begin{array}{l}\text { Fed by MANIFEST for multi- } \\
\text { object efficiency advantage }\end{array}$ & $\begin{array}{l}\text { Dependent on } \\
\text { MANIFEST } \\
\text { fiber sizes }\end{array}$ & $\begin{array}{l}\text { Dependent on } \\
\text { MANIFEST } \\
\text { fiber sizes }\end{array}$ \\
\hline
\end{tabular}

\subsection{GMACS}

The original proposal for the GMT Areal Camera and Spectrograph (GMACS) was a concept for a wide-field (9 x 18 arcmin), general purpose, medium resolution, optical, multi-object spectrograph ${ }^{8}$. The GMACS field was split across four "arms", each consisting of a two-channel spectrograph. However, that design has been de-scoped to roughly a third of the original field, in part for budgetary reasons, but also to decouple the commissioning of GMACS (a stage 1 capability) from the implementation of the wide-field corrector (a stage 3 capability) ${ }^{9}$. In doing so, GMACS becomes simpler, lower risk, faster to deliver, and has higher throughput. GMACS access to GMT's full 20 arcmin field of view will be recovered in stage 3 when both the wide-field corrector and the MANIFEST fiber feed are implemented.

GMACS is planned to be the second science instrument at GMT. GMACS has very broad "workhorse" science application, either with its slitlet masks or when using the MANIFEST fiber feed. A sample of science drivers includes the following areas:

- Time domain (GRBs, SNe, exoplanet transits) follow-up (e.g., transients sources from LSST)

- Atmospheres of brown dwarfs and exoplanets

- Dynamics of dwarf and ultra-faint dwarf galaxies

- Redshift surveys (e.g., calibration of DES, LSST photo-z)

- Formation and assembly of galaxies

- IGM (intergalactic medium) and CGM (circumgalactic medium) tomography of distant galaxies

- First light and reionization of the Universe (Lya emission-line evolution, line diagnostics of early galaxies)

To optimize the usefulness of GMACS for some of these science cases, the wavelength range currently under consideration $(370-1000 \mathrm{~nm})$ will require a red extension to $\sim 1250 \mathrm{~nm}$. Various trade studies (spectral resolution, field of view, simultaneous wavelength coverage) are underway to define the technical requirements for GMACS so that it can maximize the science return during the early years of GMT operation. 


\subsection{GMTIFS}

The design of the GMT Integral Field Spectrograph (GMTIFS) has not changed significantly since the previous conference ${ }^{10}$. Its heritage derives from the Gemini GSAOI ${ }^{11}$ and NIFS $^{12}$ instruments built by the same instrument team. The key science drivers can be addressed by the instrument's basic observational properties: it is an AO-fed YJHK (0.9$2.5 \mu \mathrm{m})$ imager (20.4 x 20.4 arcsec field) and IFU spectrograph. Although the IFU design includes four spaxel scales $(50,25,12$, and 6 mas) delivering fields of view of $4.40 \times 2.25,2.20 \times 1.13,1.06 \times 0.54$, and $0.53 \times 0.27$ arcsec, these continue to be evaluated for scientific effectiveness (e.g., is the finest scale valuable or will read noise dominate?). The IFU offers a choice between two spectral resolutions: 5,000 and 10,000.

The following key areas represent the science interests for GMTIFS:

- High spatial resolution spectroscopy of Solar System objects to examine atmospheric structure and composition

- Extend the relationship between black hole mass and galaxy velocity dispersion to greater distances and more massive black holes

- Extend the relationship between black hole mass and galaxy/cluster velocity dispersion within the local volume to the least massive black holes. This, along with the previous objective, will better anchor the relationship across the mass spectrum

- First light and reionization studies beyond the redshift range available to GMACS in Lya, as well as a selection of redder emission lines that are less extincted by dust local to the source

- Galaxy formation and dynamical evolution by IFU mapping of the galaxies across redshift

Current development activity on GMTIFS focuses on retiring the highest risks:

- Demonstrating that the very precise beam steering mirror used to track the on-instrument wavefront sensor guide star can be controlled adequately ${ }^{13}$

- Engineering the detector optimizations to minimize readout noise and provide an on-detector guide window functionality

- Fabricating large reflective camera optics

\subsection{GMTNIRS}

The GMT Near Infra-Red Spectrograph (GMTNIRS) offers an extraordinary multiplexing advantage over existing or planned high resolution IR spectrographs by providing simultaneous and full coverage in all five JHKLM bands with resolutions of 50,000 (JHK) and 100,000 (LM) ${ }^{14}$. The compact configuration (Figure 2) is achievable as a consequence of the AO feed from GMT and the implementation of silicon immersion echelle gratings.

GMTNIRS has considerable technical heritage with IGRINS ${ }^{15}$, an HK spectrograph with $\mathrm{R}=40,000$, recently completed by the same instrument team. GMTNIRS has only one configuration and only one moving part (the rotating pupil mask), and so it should be easy to use, especially in a queue observing mode, it should be highly reliable, and data reduction and analysis will be simplified.

GMTNIRS is an AO-fed instrument mounted on the folded port (Section 5.1). It has a relatively large 85 mas entrance slit, which simplifies the requirements of the on-instrument wavefront sensor while providing high throughput. The slit width choice is currently being reviewed because a smaller slit relaxes the requirements on the silicon immersion grating development, although at some cost in throughput. The production of the gratings, which represents the largest technical risk to the instrument fabrication, is the focus of current activity for this instrument. 


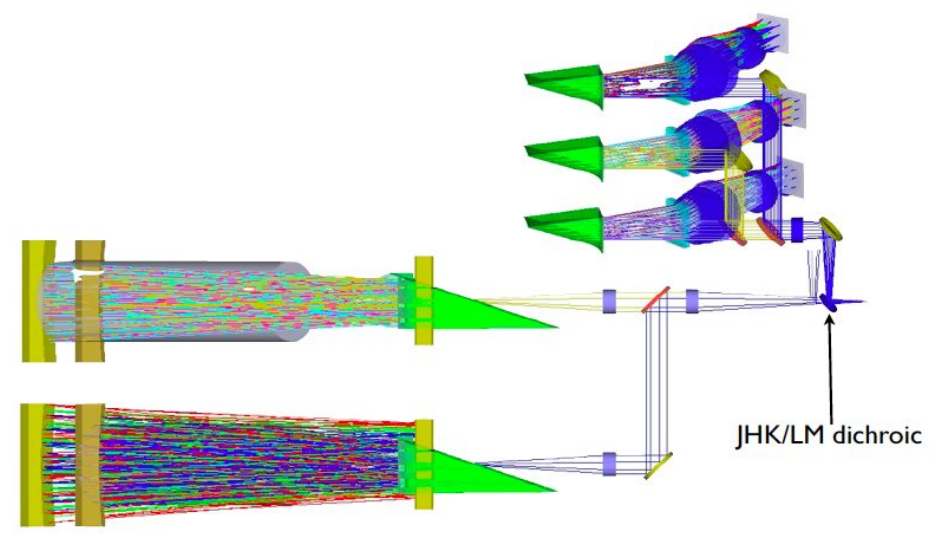

Figure 2. The GMTNIRS optical train. The beam enters the spectrograph at the right. A series of dichroics divides the signal between the 5 single-band spectrographs. In this figure, the JHK units are at the upper right and the LM units in the lower left.

Targeted science areas include:

- The formation of planetary systems and their host proto-stars

- Details of the disks surrounding young stars

- Characterization of Jupiter-mass planets

- Properties of brown dwarfs

\subsection{MANIFEST}

The Many Instrument Fiber System ${ }^{16}$ (MANIFEST) will implement Starbug robotic fiber positioners to access GMT's full 20 arcmin field of view and feed those fibers to whatever instrument is designed to accept them, significantly enhancing GMT's survey capability. In the current instrument suite, those instruments are G-CLEF and GMACS. In fact, MANIFEST can feed both instruments at the same time when the observations are coordinated, thereby dramatically raising the on-sky effectiveness of the facility by multiplexing within and across instruments. It is especially advantageous for G-CLEF, which is otherwise a single object spectrograph, providing a multiplexing gain of 4-5 with full spectral coverage, or 40 with limited coverage over a few orders. For GMACS, which already offers MOS capability, the special advantages of MANIFEST are access to the wider field, new capabilities such as small deployable IFUs, and improved spectral resolution when using fiber bundles as image slicers.

Scientifically, MANIFEST is an enabling facility. With G-CLEF, for example, MANIFEST permits velocity and chemical abundance surveys (e.g., galactic clusters, nearby dwarf galaxies) that would not be feasible for a single object spectrograph. For GMACS, MANIFEST with image slicer fiber bundles will double or triple the typical spectral resolution of $\mathrm{R} \sim 2000$ to $\mathrm{R} \sim 5000$, thereby opening new areas of science to GMACS observers.

The Starbug technology has matured considerably ${ }^{17,18,19}$ and a design for an on-sky prototype instrument is nearly complete $^{20}$. Starbugs can be placed at a center-to-center spacing of $<10 \operatorname{arcsec}$, and to a positioning accuracy of $\sim 10 \mu \mathrm{m}$ $(0.01 \mathrm{arcsec})$. Reconfiguration times will be $\sim 2-3$ minutes. MANIFEST is a stage 3 instrument.

\section{THE GMT INFRASTRUCTURE}

\subsection{Instrument mounting locations}

GMT provides three primary locations and two auxiliary locations to mount instruments. Figure 3 shows the Gregorian Instrument Rotator (GIR) Folded Port (FP) level, which is designed for three small- to moderate-sized instruments, up to $5,650 \mathrm{~kg}$, including AO sensors, and within a volume of $3.5 \times 5.5 \times 1.9-\mathrm{m}$. The FP name derives from the fact that the telescope beam is folded by the facility tertiary, located $1-\mathrm{m}$ above the rotating platform and $1.1-\mathrm{m}$ below the bottom of 
the primary mirror cells. Three instrument quadrants are available on the rotating FP platform; the fourth quadrant is reserved for utility equipment, such as the tertiary mirror (M3) deployment stage and perhaps also an instrument-specific tertiary. The facility M3 will have an IR-efficient coating, while M1 and M2 will initially be coated with aluminum. Instruments are selected by inserting/rotating M3, which is retracted to feed the instruments housed below.

Figure 4 shows the Direct Gregorian (DG) ports that are located below the FP. These are fed directly by M2 in the narrow-field mode (up to 10 arcmin diameter), or from $\mathrm{M} 2$ through a wide-field corrector and atmospheric dispersion compensator (WF/ADC) for the full 20 arcmin field. These are referred to as Direct Gregorian Narrow Field (DGNF) and Direct Gregorian Wide Field (DGWF), respectively. Four DG locations are available for instruments having sizes up to $2.8 \times 2.8 \times 5.5-\mathrm{m}$ that weigh up to $11,250 \mathrm{~kg}$ inclusive (with electronics, racks, cables). Instruments shuttle radially onto the central optical axis as needed. A set of counterweights moves to compensate for the shift in weight in order to maintain telescope balance. If a DG instrument is small enough, it may occupy a partial DG bay, providing space for an additional instrument below it. In that case, the lower instrument must include an elevator stage to raise it to the position of the focal plane.

The Gravity Invariant Station (GIS) is located on the telescope azimuth disk (Figure 3). Space is allocated for a single large instrument weighing up to $13,800 \mathrm{~kg}$. The telescope feed from the focus at the FP location to a GIS instrument is the responsibility of the instrument, and likely will be achieved either by fibers or via an optical relay.

GMT provides three additional instrument locations. These ports are not fully defined yet. Two Auxiliary Ports (AP) are located beyond the elevation bearing journals (one on either side). Instruments at these locations may provide a counterrotation to the telescope elevation in order to create a gravity invariant condition. There is also a mounting location for an instrument on the fixed portion of the Instrument Platform (IP), outside the FP (Figure 3).

The allowable volumes and masses for all instrument locations are summarized in Table 6 and Figure 5.

Table 6. The five instrument locations and the maximum masses for those instruments. Total masses for all instruments deployed at the given port locations are also shown. The maximum field of view is given. For the Direct Gregorian (DG) ports, an instrument can access either the wide-field with the corrector (WF) or the narrow field with no corrector (NF).

\begin{tabular}{|l|c|c|c|}
\hline \multicolumn{1}{|c|}{ Mount Location } & $\begin{array}{c}\text { Maximum Mass } \\
\text { per Instrument, } \\
\text { kg }\end{array}$ & $\begin{array}{c}\text { Total } \\
\text { Instrumentation } \\
\text { Mass, kg }\end{array}$ & $\begin{array}{c}\text { Field of View, } \\
\text { arcmin }\end{array}$ \\
\hline Folded Port (FP) & 5,650 & 14,550 & 3 \\
\hline Direct Gregorian (DG) & 11,250 & 45,000 & 10 (NF), 20 (WF) \\
\hline Gravity Invariant Station (GIS) & 13,800 & 13,800 & 3 \\
\hline Auxiliary Port (AP) & 3,000 & 6,000 & 3 \\
\hline Instrument Platform (IP) & 7,000 & 7,000 & 3 \\
\hline
\end{tabular}

The current suite of instruments are assigned to mount locations as follows:

- G-CLEF is at the gravity invariant station (GIS)

- GMACS and MANIFEST are at the direct Gregorian (DG)

- GMTIFS and GMTNIRS are at the folded port (FP) 


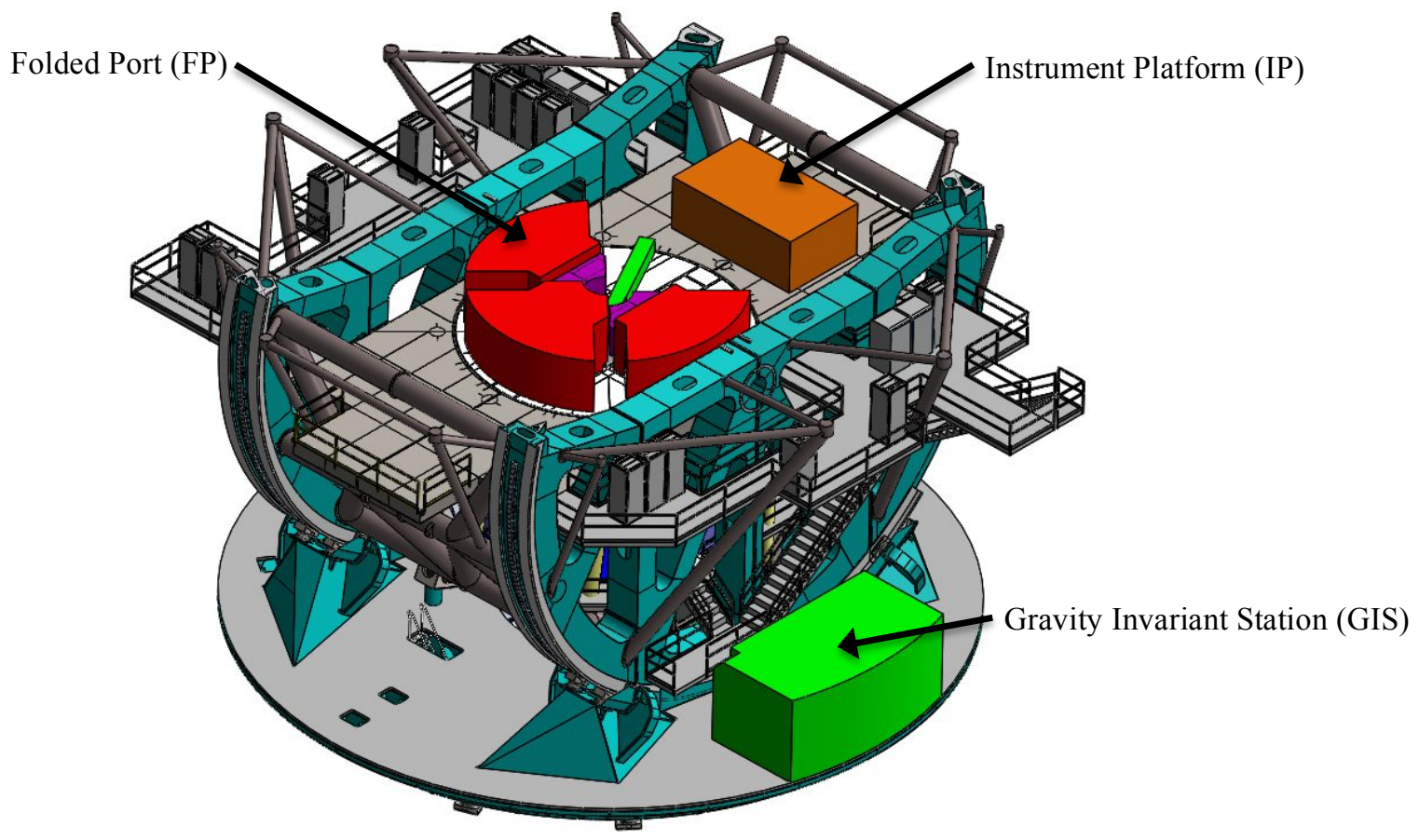

0Figure 3. Locations for mounting the FP, IP, and GIS instruments. Three FP volumes are filled as shown in red on the top of the GIR disk (white). AO-fed instruments populate two of these; the purple volumes in front of those are the Visible Wavefront Sensor Support System (VWSS). Also shown on the instrument platform is one IP instrument (brown), placed well off the rotating disk of the GIR. In addition, the gravity invariant station (GIS) volume is populated by an optical relay system (thin green rectangle on the IP rotating disk) and by a large instrument (green) on the azimuth disk.

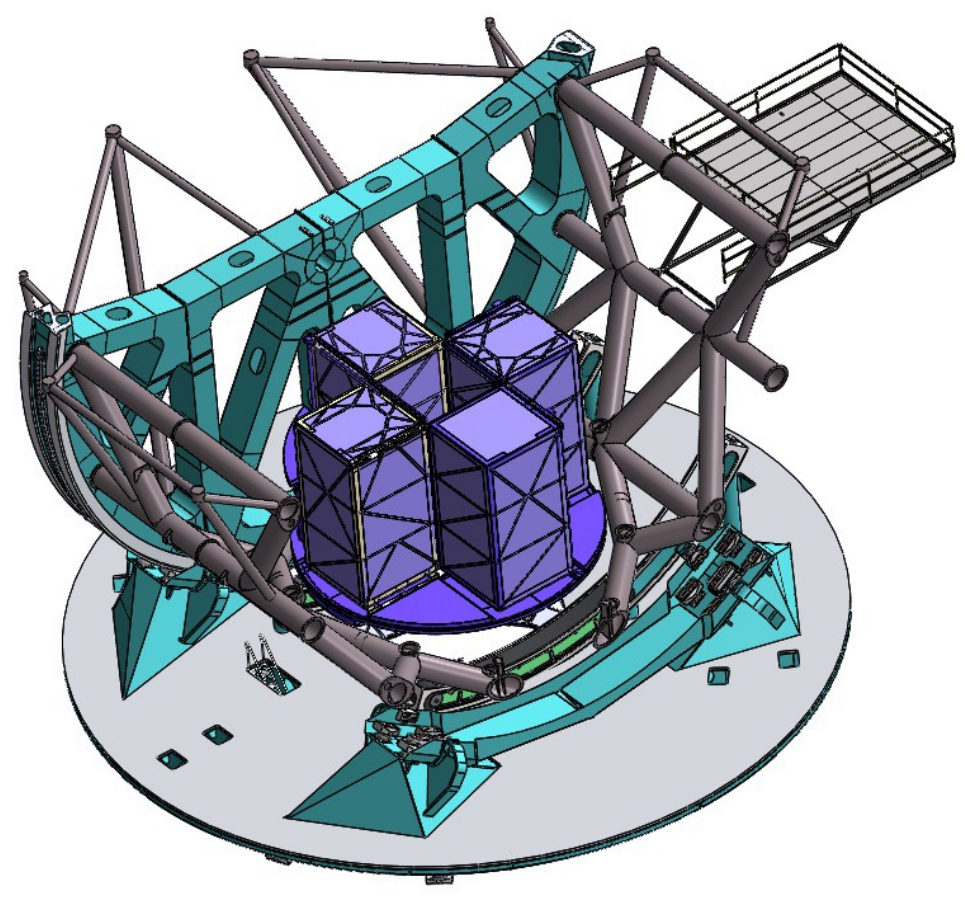

Figure 4. View of the four DG bays. This view is similar to that for the FP/IP instruments, but with the components above the GIR disk removed to see the four DG instrument volumes (blue/purple). Each large rectangle represents a full-sized instrument in its stowed position that can shuttle onto the optical axis of the telescope for observations. 


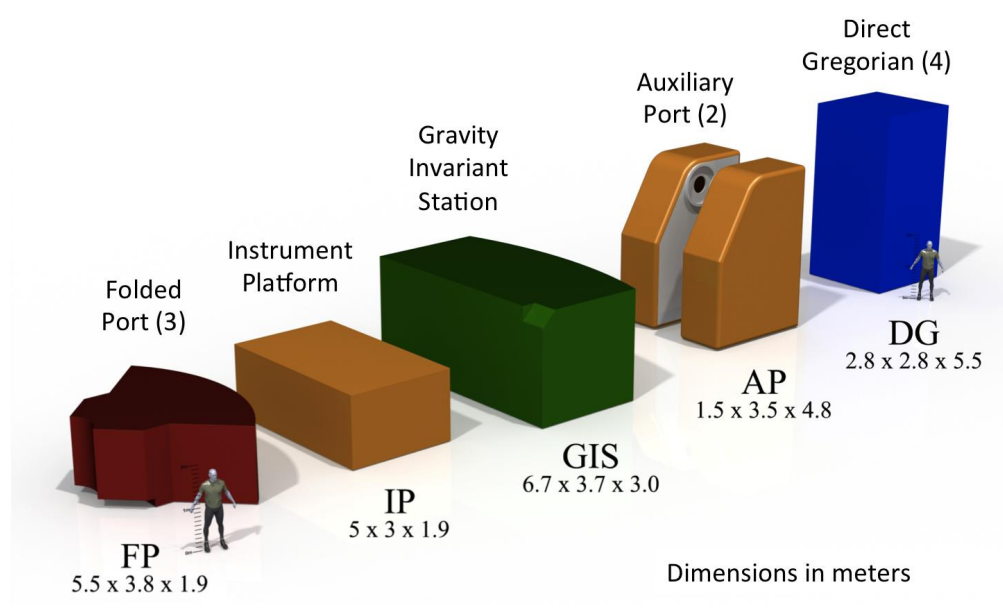

Figure 5. Allowable instrument volume for each of the mount locations. GMT has ports for 11 live instruments.

\subsection{Adaptive optics (AO)}

GMT delivers AO-corrected images ${ }^{21}$ to the instruments installed at the folded ports provided that the instrument complies with the wavefront sensor requirements. These include:

1. A dichroic window to the instrument, tilted to direct visible light shortward of $920 \mathrm{~nm}$ toward the sensors in items 2 and 3 below.

2. An external optical natural guide star wavefront sensor that is also required for natural guide star AO (NGSAO)

3. An external optical laser guide star wavefront sensor, required for laser tomography AO (LTAO)

4. An internal infrared on-instrument wavefront sensor (OIWFS) to measure low-order aberrations (tip-tilt, focus, and truth wavefront error) introduced by instrument flexure and non-common paths effects

Items 1-3 are shown in Figure 6 attached to a mechanical support that mounts to the front of an AO instrument.
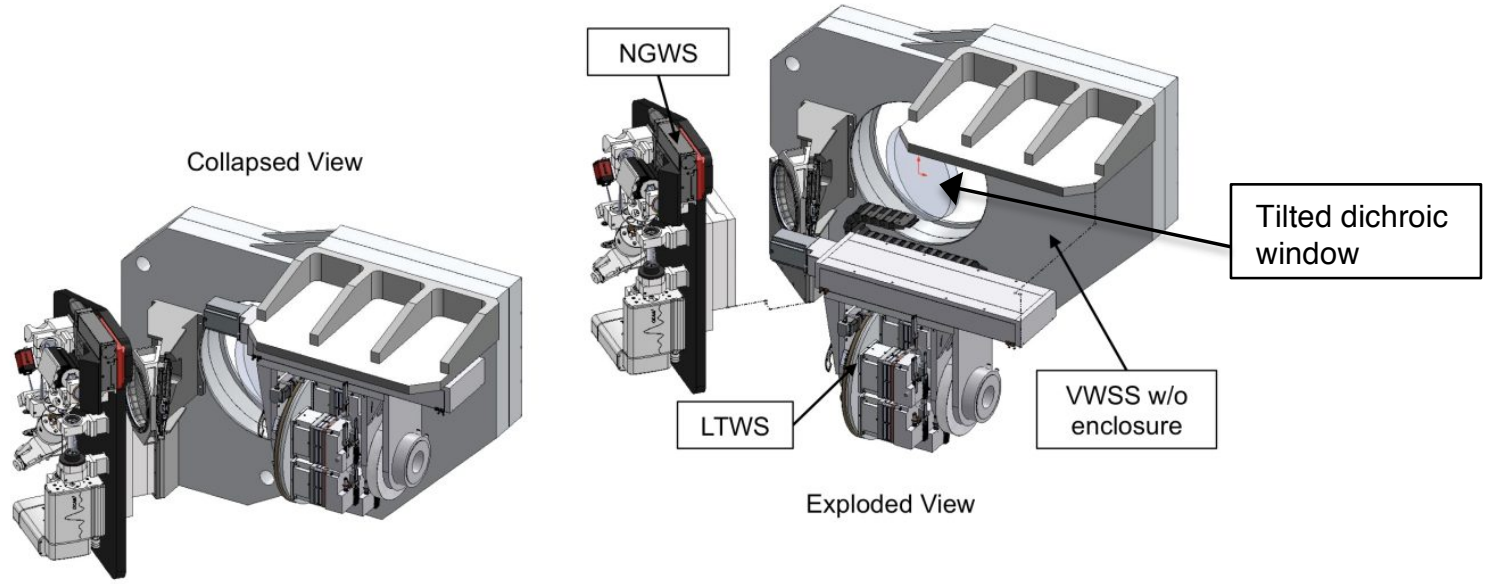

Exploded View

Figure 6. The Visible Wavefront Sensor System (VWS). The VWS is mounted in front of each AO-fed instrument to provide wavefront sensors for natural guide star AO using the NGWS sensors, and laser tomography AO, using the LTWS sensors in addition. The Visible Wavefront Sensor Support System (VWSS) is the mechanical interface to the instrument. The tilted window $(20 \mathrm{deg})$ is seen in the exploded view on the right. 
In addition to NGSAO and LTAO, GMT supports a ground layer AO (GLAO) mode to provide partial atmospheric correction over fields up to the full 20 arcmin diameter. The smaller the field, though, the better the correction. In practice, GLAO is most effective for fields of a few arcmin. GLAO at GMT relies only on natural guide stars and therefore is always available when the adaptive secondary mirrors are operational. Natural seeing instruments need no additional hardware or software. However, to realize the benefits of GLAO, instruments must be designed for the higher performance image quality. Figure 7 illustrates the expected PSF and throughput improvements from GLAO as a function of field size and observing wavelength.
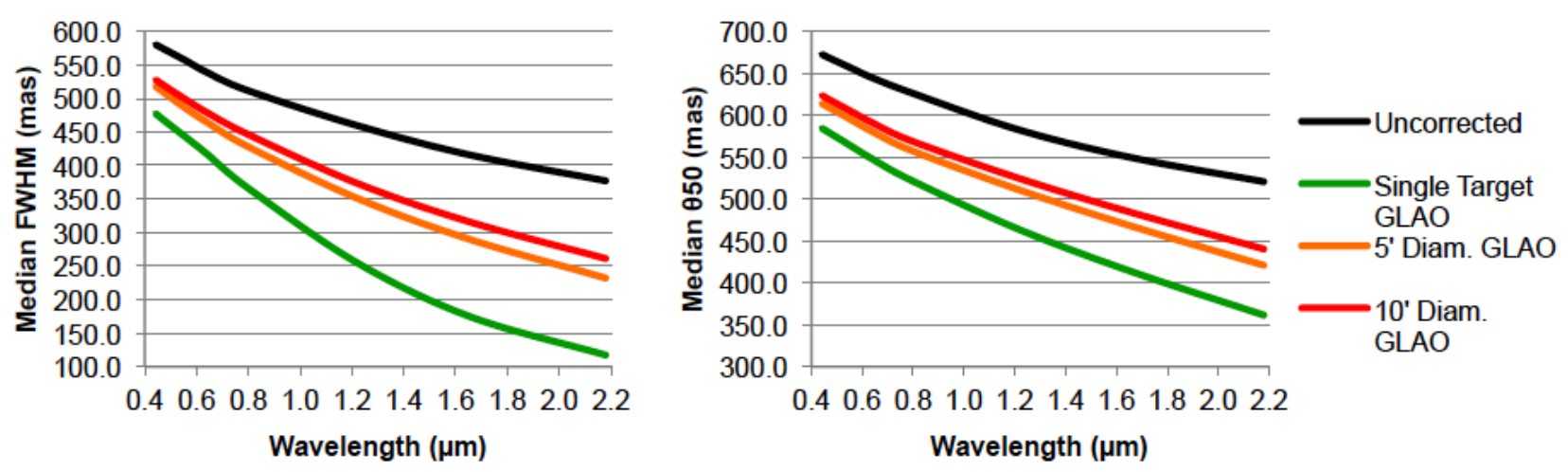

Figure 7. (Left) Mean FWHM across the corrected field of view, versus wavelength for different corrected field sizes (and uncorrected). (Right) Mean 50\% encircled energy diameter versus wavelength. Simulations represent a median star field at the galactic pole. Performance will typically be better as one observes closer to the galactic plane.

\subsection{Switching Between Instruments}

Depending on the desired instrument port, the mode of transitioning between instruments is selected according to the steps summarized in Table 7. The timing assumes that the telescope is at a zenith distance of $30 \mathrm{deg}$ when the changeover begins to account for the time to move to zenith when necessary (e.g., in order to move M3 or the Corrector$\mathrm{ADC}$ in to, or out of, position). The total changeover time begins when the telescope starts its moves to zenith and ends when the instrument is in place. The time to move to a new position away from zenith is not included.

Table 7. Procedures and times to switch between instruments

\begin{tabular}{|l|l|l|l|}
\hline $\begin{array}{c}\text { Current } \\
\text { Instrument } \\
\text { Port }\end{array}$ & $\begin{array}{l}\text { Desired } \\
\text { Instrument } \\
\text { Port }\end{array}$ & \multicolumn{1}{|c|}{ Operational Steps } & \multicolumn{1}{|c|}{$\begin{array}{c}\text { Time to Change (min), } \\
\text { Nominal [Goal] }\end{array}$} \\
\hline \multirow{4}{*}{ FP } & FP & Rotate M3 & $3[2]$ \\
\cline { 2 - 4 } & AP, IP & Rotate M3; Rotate GIR & $3[2]$ \\
\cline { 2 - 4 } & GIS & $\begin{array}{l}\text { Depending on instrument pickoff requirement, } \\
\text { rotate M3; } \\
\text { or, Go to zenith; retract M3; deploy M3-special } \\
\text { if needed }\end{array}$ & $\begin{array}{l}3[2] \\
10.75[6.75]= \\
0.75+5[3]+5[3]\end{array}$ \\
\cline { 2 - 5 } & DG & $\begin{array}{l}\text { Go to zenith; retract M3 } \\
\text { (assumes desired DG instrument is already } \\
\text { deployed on-axis) }\end{array}$ & $\begin{array}{l}5.75[3.75]= \\
0.75+5[3]\end{array}$ \\
\hline \multirow{2}{*}{ DG } & DG & $\begin{array}{l}\text { Go to zenith; retract current instrument into bay; } \\
\text { deploy new instrument to on-axis position. This } \\
\text { change must be performed during the day. }\end{array}$ & 60 \\
\hline DGNF & DGWF & $\begin{array}{l}\text { Go to zenith; Insert Corrector/ADC. } \\
\text { If a different instrument is required, then the } \\
\text { DG-DG change is also required. }\end{array}$ & $\begin{array}{l}5.75[3.75]= \\
0.75+5[3]\end{array}$ \\
\hline
\end{tabular}


The time to perform an instrument change in the opposite direction is the same since the same processes are involved. These timings do not include the time, if needed, to adjust the active optics as this is considered a part of the normal move to a new object.

\subsection{Utilities and Work Space}

All instruments will be provided with the required utilities that were identified at the time of their conceptual designs, including AC mains and UPS power, ethernet ports, cryo-cooler lines, dry nitrogen/air, helium compressor lines, compressed air, and a chilled liquid to remove excess heat. Instrument lifts are available for installation.

The nearby Summit Support Building (Figure 8) provides clean space for instrument assembly, testing, repairs, and upgrades. Sufficient room is provided to allow concurrent activities on two of the largest instruments. The same utilities are available in the instrument bays as those provided on the telescope. In addition, clean rooms, an electronic lab, and a detector lab are available for critical disassembly or assembly.

Instruments are transported between the telescope enclosure and the Summit Support Building on the same cart that is used to transport the primary mirror segments for re-coating. The cart is configured with standard mounts to accommodate the instruments, and driven from the instrument bays to a position under the enclosure overhead crane.

\section{CONCLUSIONS}

The GMTO instrumentation program has now been implemented, with five instrument teams under contract to advance their instrument developments. The first science instruments, G-CLEF and GMACS, will operate in the natural seeing mode, perhaps with only four or five primary mirror segments in stage 1 of the GMT commissioning plan. These instruments are progressing on schedule for delivery at a time when the GMT facility is ready for them. The AO instruments follow in stages 2 (GMTIFS) and 3 (GMTNIRS). These instruments are somewhat more complex, as expected to meet their high performance AO image quality specifications, and consequently the longer lead times are commensurate with their schedule. MANIFEST, also a stage 3 instrument, is completing a suite of prototyping activities to reduce later risk. Its prototyping also leads to a scientifically valuable on-sky demonstrator of the Starbug concept within 1-2 years.

Since the last SPIE meeting, considerable effort has been focused on developing the requirements flow down from science drivers to instrument definition (functional, technical, operational requirements). These activities are nearing completion for most instruments.

The instrument development roadmap that was presented at the previous meeting is now in an active phase. The first generation suite of GMT instruments enables a broad range of science applications that take advantage of GMT's wide field of view and large aperture to provide excellent survey capabilities, and are well suited for both bright- and darktime, excellent and fair atmospheric conditions, as well as natural seeing and high order AO modes. GMT will soon begin the process of identifying and developing subsequent generations of instruments that will be funded primarily through the operations budget. 


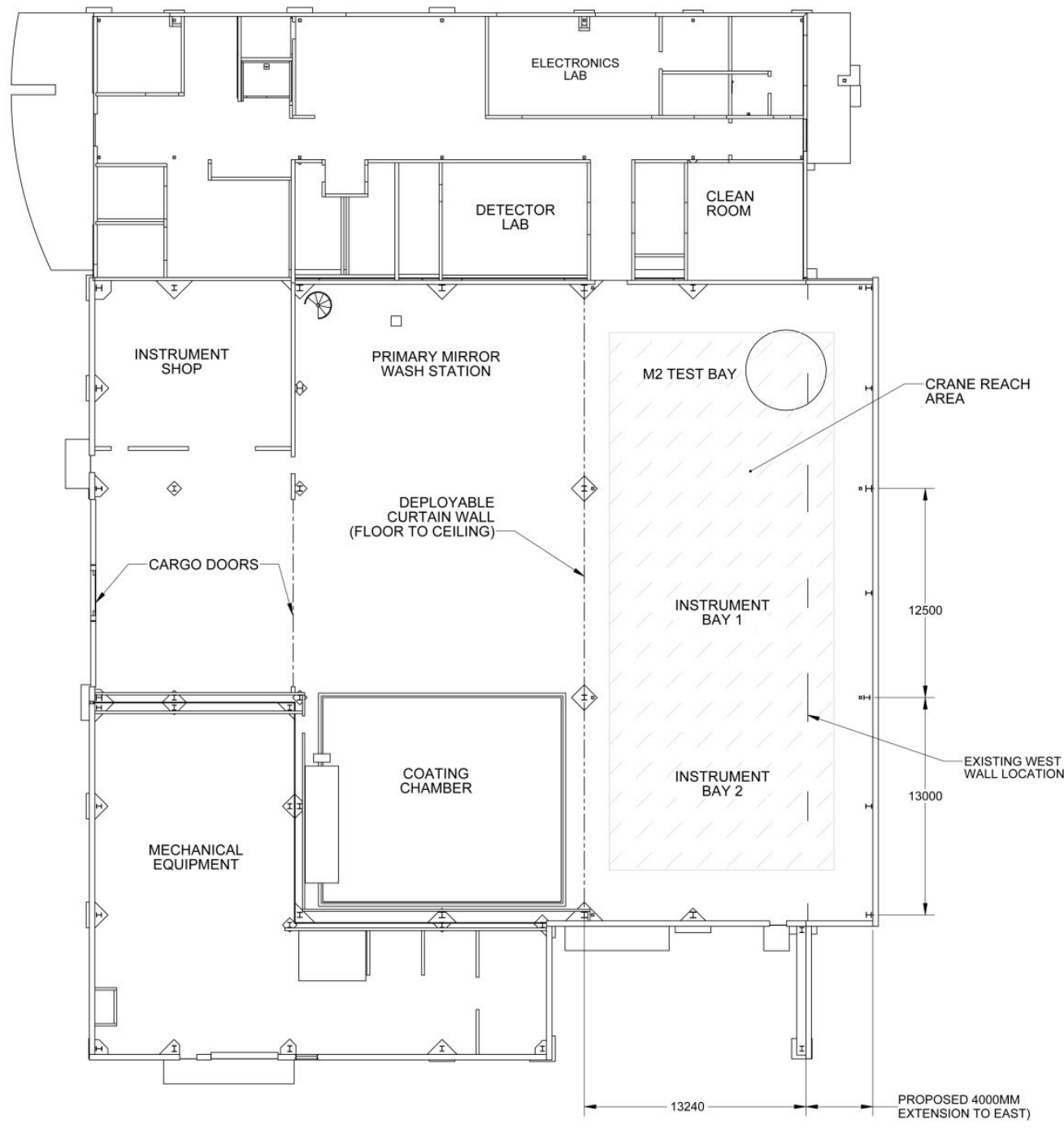

Figure 8. The layout of the Summit Support Building, about $40 \mathrm{~m}$ from the GMT enclosure. Note the two large instrument bays to the right that are kept clean to better than class 100,000 . These can be subdivided by movable tents for tighter cleanliness if needed. Or, subcomponents of instruments can be moved to the smaller clean room or detector lab toward the top of the figure.

\section{ACKNOWLEDGEMENTS}

This work has been supported by the GMTO Corporation, a non-profit organization operated on behalf of an international consortium of universities and institutions: Astronomy Australia Ltd, the Australian National University, the Carnegie Institution for Science, Harvard University, the Korea Astronomy and Space Science Institute, the Smithsonian Institution, The University of Texas at Austin, Texas A\&M University, University of Arizona and University of Chicago. This work has also been supported in part by the National Science Foundation under Scientific Program No. 10 as issued for support of the Giant Segmented Mirror Telescope for the United States Astronomical Community, in accordance with Proposal No. AST-0443999 submitted by AURA. 


\section{REFERENCES}

[1] Jacoby, G. H.; Bouchez, A., Colless, M., DePoy, D., Fabricant, D., Hinz, P., Jaffe, D., Johns, M., McCarthy, P., McGregor, P., Shectman, S., Szentgyorgyi, A., "The instrument development and selection process for the Giant Magellan Telescope," Proc. SPIE 8446, 1GJ, 1 (2012).

[2] Bernstein, R. A., McCarthy, P. J., Raybould, K., Bigelow, B. C., Bouchez, A. H., Filgueira, J. M., Gunnels, S., Jacoby, G. H., Johns, M., Sawyer, D., Sheehan, M., "Overview and status of the Giant Magellan Telescope project," Proc. SPIE 9145, 47 (2014).

[3] Johns, M., et al., "Design of the Giant Magellan Telescope," Proc. SPIE 9145, 50-50 (2014).

[4] Szentgyorgyi, A. et al., "A Preliminary Design for the GMT-Consortium Large Earth Finder (G-CLEF)," Proc. SPIE 9147, 78-78 (2014).

[5] Keller, S., Bessell, M.S., Frebel, A., Casey, A.R., Asplund, M., Jacobson, H.R., Lind, K., Norris, J.E., Yong, D., Heber, A., Magic, Z., da Costa, G.S., Schmidt, B.P. and Tisserand, P., "A single low-energy, iron-poor supernova as the source of metals in the star SMSS J0313000.36-670839.3," Nature 7489, 463 (2014).

[6] Rodler, F. and Lopez-Morales, M., "Feasibility studies for the detection of O2 in an Earth-like exoplanet," ApJ 781, 12 (2014).

[7] Furesz, G., et al., "The G-CLEF spectrograph optical design: an update to the white pupil echelle configuration," Proc. SPIE 9147, 343-343 (2014).

[8] DePoy, D. L., Marshall, J. L., Prochaska, T., Behm, T. W., Smee, S. A., Barkhouser, R. H., Hammond, R. P., Shectman, S. A., Papovich, C., "The GMACS spectrograph for the Giant Magellan Telescope," Proc. SPIE 8446, 292292 (2012).

[9] DePoy, D. L., Allen, R., Li, T., Marshall, J. L., Papovich, C., Prochaska, T., and Schectman, S., "An Update on the Wide Field, Multi-Object, Moderate-Resolution, Spectrograph for the Giant Magellan Telescope," Proc. SPIE 9147, $72-$ 72 (2014).

[10] McGregor, P. J., Bloxham, G. J., Boz, R., Davies, J., Doolan, M. C., Ellis, M., Hart, J., Nielsen, J. J., Parcell, S., Sharp, R. G. and Stevanovic, D., "The GMT integral-field spectrograph (GMTIFS) conceptual design," Proc. SPIE 8446, 1-1 (2012).

[11] McGregor, P. J., Hart, J., Stevanovic, D., Bloxham, G., Jones, D., Van Harmelen, J., Griesbach, J., Dawson, M., Young, P. and Jarnyk, M. A., "Gemini South Adaptive Optics Imager (GSAOI)," Proc. SPIE 5492, 1033-1044 (2004).

[12] McGregor, P. J., Hart, J., Conroy, P. G., Pfitzner, M. L., Bloxham, G. J., Jones, D. J., Downing, M. D., Dawson, M., Young, P., Jarnyk, M. and Van Harmelen, J., "Gemini near-infrared integral field spectrograph (NIFS)," Proc. SPIE 4841, 1581-1591 (2003).

[13] Sharp, R., Boz, R., Hart, J., Bloxham, G., Bundy, D., Davis, J., Vest, M., and Young, P. J., "The adaptive optics beam steering mirror for the GMT Integral-Field Spectrograph, GMTIFS," Proc. SPIE 9151, 11-11 (2014).

[14] Jaffe, D. T., Barnes, S. I., Brooks, C. B., Gully-Santiago, M., Pak, S., Park, C., Yuk, I.-S."GMTNIRS (Giant Magellan telescope near-infrared spectrograph): optimizing the design for maximum science productivity and minimum risk," Proc. SPIE 9147, 74-74 (2014).

[15] Park, C. P., et al., "Design and early performance of IGRINS (immersion grating infrared spectrometer)," Proc. SPIE 9147, 48-48 (2014). 
[16] Lawrence, J., et al., "The MANIFEST fibre positioning system for the Giant Magellan telescope," Proc. SPIE 9147, 341-341 (2014).

[17] Brown, D. M., et al., "Starbug fiber positioning robots: performance and reliability enhancements," Proc. SPIE 9151, 45-45 (2014).

[18] Piersiak, R. P., Goodwin, M., Gilbert, J., and Muller, R., "Automating the measurement and characterization of piezoelectric tubes for Starbugs," Proc. SPIE 9147, 357-357 (2014).

[19] Goodwin, M., Lorente, N. P. F., Hong, S. E., and Satorre, C., "Field target allocation and routing algorithms for Starbugs," Proc. SPIE 9152, 26-26 (2014).

[20] Kuehn, K., et al., "TAIPAN: optical spectroscopy with StarBugs," Proc. SPIE 9147, 35-35 (2014).

[21] Bouchez, A., "The Giant Magellan telescope adaptive optics program," Proc. SPIE 9148, 31-31 (2014). 